\title{
POLYNOMIAL FIRST INTEGRALS FOR THE CHEN AND LÜ SYSTEMS
}

\author{
JAUME LLIBRE \\ Departament de Matemàtiques \\ Universitat Autònoma de Barcelona, 08193, Bellaterra, Barcelona, \\ Catalonia, Spain, e-mail: jllibre@mat.uab.es \\ CLAUDIA VALLS \\ Departamento de Matemática \\ Instituto Superior Técnico (U.T.L.). Av. Rovisco Pais 1049-001 Lisboa, \\ Portugal,e-mail:cvalls@math.ist.utl.pt
}

June 27, 2011

We characterize all the values of the parameters for which the Chen and Lü systems have polynomial first integrals by using weight homogeneous polynomials and the method of characteristics for solving partial differential equations. We improve previous results which were not complete.

\section{Introduction and statement of the main results}

The following real differential system

$$
\begin{aligned}
& \dot{x}=a(y-x), \\
& \dot{y}=(c-a) x-x z+c y, \\
& \dot{z}=x y-b z,
\end{aligned}
$$

where $a, b, c \in \mathbb{R}$ are parameters is known as the Chen system [Chen \& Ueta, 1999]. It exhibits chaotic phenomena which resembles some familiar features from both the Lorenz and the Rössler attractors, for suitable choices of the parameters. Despite of its similar structure to the Lorenz system, it is not topologically equivalent. This is why Lü and Chen investigated the real differential system

$$
\begin{aligned}
& \dot{x}=a(y-x), \\
& \dot{y}=-x z+c y, \\
& \dot{z}=x y-b z,
\end{aligned}
$$

where $a, b, c \in \mathbb{R}$ are parameters, which now is usually called the Lü system [Lü \& Chen, 2002]. The Lü system connects the Lorenz system and the Chen system and represents a transition from one to the other. For more details see [Lü \& Chen, 2002]. Moreover, recently Lü and Zhang [Lü \& Zhang, 2007] and Lü [Lü, 2009] characterize the invariant algebraic surfaces of the Chen and of the Lü systems, respectively. Furthermore, Lü in [Lü, 2007] characterize the Darboux first integrals of the Chen system. These recent years the dynamics of the Chen system has been analyzed from many different points of view. See for instance [Bashkirtseva et al., 2010, Cafagna \& Grassi, 2008,

Cai et al., 2009, Cao et al., 2008,

Chen \& Wang, 2007, Chen \& Zhou, 2009, Chowdhury \& Hashim, 2009, Denquan \& Zhixiang, 2009, Fallahi et al., 2008, 
Hou et al., 2008,

Mahmoud et al., 2007, Yao \& Liu, 2010].

The vector field associated to (1) is

$X=a(y-x) \frac{\partial}{\partial x}+((c-a) x-x z+c y) \frac{\partial}{\partial x}+(x y-b z) \frac{\partial}{\partial x}$,

and the vector field associated to (2) is

$$
X=a(y-x) \frac{\partial}{\partial x}+(-x z+c y) \frac{\partial}{\partial x}+(x y-b z) \frac{\partial}{\partial x} .
$$

Let $U$ be an open subset of $\mathbb{R}^{3}$ such that $\mathbb{R}^{3} \backslash U$ has zero Lebesgue measure. We say that a real function $H=H(x, y, z): U \subset \mathbb{R}^{3} \rightarrow \mathbb{R}$ is a first integral if $H(x(t), y(t), z(t))$ is constant for all values of the solution $(x(t), y(t), z(t))$ of $X$ where it is defined, i.e., if $X H=0$.

Two functions $f_{1}(x, y, z)$ and $f_{2}(x, y, z)$ are independent if their gradients are linearly independent vectors for all $(x, y, z) \in \mathbb{R}^{3}$ except perhaps for a set of zero Lebesgue measure.

If the vector field $X$ has two independent first integrals $H_{1}$ and $H_{2}$, we say that it is completely integrable. In this case the orbits of $X$ are contained in the curves $\left\{H_{1}(x, x, y, z)=h_{1}\right\} \cap\left\{H_{2}(x, y, z)=\right.$ $\left.h_{2}\right\}$, when $h_{1}$ and $h_{2}$ vary in $\mathbb{R}$.

When $a=0$ the Chen and Lü differential systems are completely integrable as it is shown in the following result. Its proof is tedious but it follows easily from direct computations.

Theorem 1.1. When $a=0$ the Chen and Lü systems are integrable with the first integrals $H_{1}=x$ and $\mathrm{H}_{2}$.

(a) For the Chen system

$$
\begin{aligned}
H_{2}= & x \exp \left(\frac{2(b-c) \arctan \left(\frac{b-c+\frac{2 A}{b z-x y}}{\sqrt{4 B-(b-c)^{2}}}\right)}{\sqrt{4 B-(b-c)^{2}}}\right) \\
& \left(B\left(x y^{2}+x z^{2}-c x z-(b+c) y z\right)+\right. \\
& \left.c(c-b) x^{2} y+c x\left(b^{2} z-x^{2} z+c x^{2}\right)\right)
\end{aligned}
$$

where $A=c\left(x^{2}+b z\right)-x^{2} z$ and $B=x^{2}-b c$.

(b) For the Lü system

$$
\begin{aligned}
H_{2}= & z \exp \left(\frac{-(b-c) \arctan \left(\frac{2 x y-(b+c) z}{z \sqrt{4 x^{2}-(b+c)^{2}}}\right)}{\sqrt{4 x^{2}-(b+c)^{2}}}\right) \\
& \sqrt{x+\frac{x y^{2}}{z^{2}}-\frac{(b+c) y}{z}} .
\end{aligned}
$$

In view of Theorem 1.1 from now on we consider only the case $a \neq 0$ in both the Chen and the Lü systems.

Our main result for the Chen and Lü systems are the following two theorems.

Theorem 1.2. The Chen differential system with $a \neq 0$ has a polynomial first integral if and only if $b=c=0$. In this case any polynomial first integral is a polynomial in the variable $y^{2}+z^{2}+2 a z$.

Theorem 1.2 provides a counterexample to statement (b) of Theorem 1 of [Lü, 2007], where the author claims that the Chen system has no Darboux polynomial first integrals and of course the polynomial first integral $y^{2}+z^{2}+2 a z$ is a Darboux polynomial for the Chen system.

Theorem 1.3. The Lü system with $a \neq 0$ has $a$ polynomial first integral if and only if $b=c=0$. In this case any polynomial first integral is a polynomial in the variable $y^{2}+z^{2}$.

The result of Theorem 1.3 is well known, see [Llibre \& Zhang, 2002, Lü, 2009].

The proof of Theorem 1.2 is given in section 2 , but we only provide an sketch of the proof of Theorem 1.3 in section 3 . We note that in the proof of Theorems 1.2 we use similar arguments to the ones used in the papers [Lü \& Zhang, 2007] and [Lü, 2009].

\section{Proof of Theorem $\mathbf{1 . 2}$}

In this section we prove Theorem 1.2. For simplifying the computations we introduce the following weight change of variables

$$
x=\mu^{-1} X, \quad y=\mu^{-2} Y, \quad z=\mu^{-2} Z, \quad t=\mu T,
$$

with $\mu \in \mathbb{R} \backslash\{0\}$. Then the Chen system (1) becomes

$$
\begin{aligned}
& X^{\prime}=a(Y-\mu X), \\
& Y^{\prime}=-X Z+\mu c Y+\mu^{2}(c-a) X, \\
& Z^{\prime}=X Y-\mu b Z,
\end{aligned}
$$

where the prime denotes the derivative of the variables with respect to $T$.

A polynomial $G(X, Y, Z)$ is said to be weight homogeneous of degree $m \in \mathbb{N}$ with respect to the 
weight exponent $s=\left(s_{1}, s_{2}, s_{3}\right)$ for all $\mu \in \mathbb{R} \backslash\{0\}$ we have

$$
G\left(\mu^{s_{1}} X, \mu^{s_{2}} Y, \mu^{s_{3}} Z\right)=\mu^{m} G(X, Y, Z) .
$$

Here $\mathbb{N}$ is the set of positive integers.

Let $g(x, y, z)$ be a polynomial first integral of system (1). Set

$$
\begin{aligned}
G(X, Y, Z) & =\mu^{m} g\left(\mu^{-1} X, \mu^{-2} Y, \mu^{-2} Z\right) \\
& =\sum_{i=0}^{m} \mu^{i} G_{i}(X, Y, Z),
\end{aligned}
$$

where $G_{i}$ is the weight homogeneous part with weight degree- $m-i$ of $G$, and $m$ is the weight degree of $G$ with the weight exponent $s=(1,2,2)$.

From the definition of a polynomial first integral we have

$$
\begin{aligned}
& a(y-\mu x) \sum_{i=0}^{m} \mu^{i} \frac{\partial G_{i}}{\partial x}+ \\
& \left(-x z+\mu c y+\mu^{2}(c-a) x\right) \sum_{i=0}^{m} \mu^{i} \frac{\partial G_{i}}{\partial y}+ \\
& (x y-\mu b z) \sum_{i=0}^{m} \mu^{i} \frac{\partial G_{i}}{\partial z}=0
\end{aligned}
$$

where we still use $x, y, z$ instead of $X, Y, Z$.

Equating in (5) the terms with $\mu^{i}$ for $i=$ $0,1, \ldots, m+2$, we get

$$
\begin{aligned}
L\left[G_{0}\right]= & 0, \\
L\left[G_{1}\right]= & a x \frac{\partial G_{0}}{\partial x}-c y \frac{\partial G_{0}}{\partial y}+b z \frac{\partial G_{0}}{\partial z}, \\
L\left[G_{j}\right]= & a x \frac{\partial G_{j-1}}{\partial x}-c y \frac{\partial G_{j-1}}{\partial y}+ \\
& b z \frac{\partial G_{j-1}}{\partial z}-(c-a) x \frac{\partial G_{j-2}}{\partial y},
\end{aligned}
$$

for $j=2,3, \ldots, m+2$, where $G_{j}=0$ for $j>m$ and $L$ is the linear partial differential operator of the form

$$
L=a y \frac{\partial}{\partial x}-x z \frac{\partial}{\partial y}+x y \frac{\partial}{\partial z} .
$$

The characteristic equations associated to the linear partial differential operator are

$$
\frac{d x}{d z}=\frac{a}{x}, \quad \frac{d y}{d z}=-\frac{z}{y} .
$$

This system has the general solution

$$
x^{2}-2 a z=d_{1}, \quad y^{2}+z^{2}=d_{2},
$$

where $d_{1}$ and $d_{2}$ are constants of integration. According to this, we make the change of variables

$$
u=x^{2}-2 a z, \quad v=y^{2}+z^{2}, \quad w=z .
$$

Its inverse transformation is

$$
x= \pm \sqrt{u+2 a w}, \quad y= \pm \sqrt{v-w^{2}}, \quad z=w .
$$

In the following, for simplicity, we only consider the case $x=\sqrt{u+2 a w}, y=\sqrt{v-w^{2}}, z=w$. Under the changes of variables (8) and (9), the first equation of (6) becomes the following ordinary differential equation (for fixed $u, v$ ):

$$
\sqrt{u+2 a w} \sqrt{v-w^{2}} \frac{d \bar{G}_{0}}{d w}=0,
$$

where $\bar{G}_{0}$ is $G_{0}$ written in the variables $u, v$ and $w$. In what follows we always use the notation $\bar{\theta}$ to denote $\theta(x, y, z)$ written in the variables $u, v, w$. The last equation has the following general solution $\bar{G}_{0}=\bar{T}_{0}(u, v)$, where $\bar{T}_{0}$ is an arbitrary smooth function in $u$ and $v$. So,

$$
G_{0}(x, y, z)=\bar{G}_{0}(u, v, w)=T_{0}\left(x^{2}-2 a z, y^{2}+z^{2}\right) .
$$

Since $G_{0}$ is a weight homogeneous polynomial and the weight degrees of $u$ and $v$ in the variables $x, y, z$ are 2 and 4 , respectively, $G_{0}$ should have weight degree either $m=4 n$, or $m=4 n-2$ for some convenient $n \in \mathbb{N}$. So $G_{0}$ has the form

$$
G_{0}=\sum_{i=0}^{n} a_{i}\left(x^{2}-2 a z\right)^{2 i}\left(y^{2}+z^{2}\right)^{n-i},
$$

with the weight degree $4 n$, or

$$
G_{0}=\sum_{i=1}^{n} a_{i}\left(x^{2}-2 a z\right)^{2 i-1}\left(y^{2}+z^{2}\right)^{n-i},
$$

with the weight degree $4 n-2$.

Case 1: $G_{0}$ has the form (11). Substituting $G_{0}$ into the second equation of (6), we can prove that

$$
\begin{aligned}
L\left[G_{1}\right]= & \sum_{i=1}^{n}[2 a(2 i-1)-2 c(n-i)] \\
& a_{i}\left(x^{2}-2 a z\right)^{2 i-1}\left(y^{2}+z^{2}\right)^{n-i} \\
& +\sum_{i=1}^{n}\left[\left(4 a^{2}-2 a b\right)(2 i-1)\right] \\
& a_{i}\left(x^{2}-2 a z\right)^{2 i-2}\left(y^{2}+z^{2}\right)^{n-i} z \\
& +\sum_{i=1}^{n} 2(b+c)(n-i) \\
& a_{i}\left(x^{2}-2 a z\right)^{2 i-1}\left(y^{2}+z^{2}\right)^{n-i-1} z^{2} .
\end{aligned}
$$


Using the transformations (8) and (9) and working in a similar way to solve $\bar{G}_{0}$ we get the following ordinary differential equation (for fixed $u$ and $v$ ):

$$
\begin{aligned}
& \sqrt{u+2 a w} \sqrt{v-w^{2}} \frac{d \bar{G}_{1}}{d w}= \\
& \sum_{i=1}^{n}[2 a(2 i-1)-2 c(n-i)] a_{i} u^{2 i-1} v^{n-i} \\
& +\sum_{i=1}^{n}\left[\left(4 a^{2}-2 a b\right)(2 i-1)\right] a_{i} u^{2 i-2} v^{n-i} w \\
& +\sum_{i=1}^{n} 2(b+c)(n-i) a_{i} u^{2 i-1} v^{n-i-1} w^{2} .
\end{aligned}
$$

Using

$$
\frac{d}{d w}\left(\sqrt{u+2 a w} \sqrt{v-w^{2}}\right)=\frac{-u w+a\left(v-3 w^{2}\right)}{\sqrt{u+2 a w} \sqrt{v-w^{2}}},
$$

it is easy to deduce that the integration of the previous equation with respect to $w$ is

$$
\begin{aligned}
\bar{G}_{1}= & \sum_{i=1}^{n} \frac{1}{a}[2 a(2 i-1)-2 c(n-i)] \\
& a_{i} u^{2 i-1} v^{n-i-1} \sqrt{u+2 a w} \sqrt{v-w^{2}} \\
& +\sum_{i=0}^{n}\left[\left(4 a^{2}-2 a b\right)(2 i+1) a_{i+1}\right. \\
& \left.+(2 a(2 i-1)-2 c(n-i)) a_{i}\right] u^{2 i} v^{n-i-1} . \\
& \cdot \int_{n}^{n} \frac{w d w}{\sqrt{u+2 a w} \sqrt{v-w^{2}}} \\
& +\sum_{i=1}^{n}\left[2(b+c)(n-i)+\frac{3}{a}(2 a(2 i-1)\right. \\
& -2 c(n-i))] a_{i} u^{2 i-1} v^{n-i-1} . \\
& \cdot \int \frac{w^{2} d w}{\sqrt{u+2 a w} \sqrt{v-w^{2}}}+\bar{T}_{1}(u, v),
\end{aligned}
$$

where $\bar{T}_{1}(u, v)$ is an arbitrary smooth function in $u$ and $v$. Since $\bar{G}_{1}$ is a weight homogeneous polynomial of weight degree $4 n-3$, we must have $\bar{T}_{1}(u, v)=0$, and

$$
\begin{aligned}
& \left(4 a^{2}-2 a b\right)(2 i+1) a_{i+1}+ \\
& (2 a(2 i-1)-2 c(n-i)) a_{i}=0, \\
& i=0, \ldots, n, \\
& \left(2(b+c)(n-i)+\frac{3}{a}(2 a(2 i-1)-\right. \\
& 2 c(n-i))) a_{i}=0, \\
& i=1, \ldots, n,
\end{aligned}
$$

where $a_{0}=a_{n+1}=0$. It is easy to prove that since $a \neq 0$, conditions (12) are equivalent to one of the following conditions, either $b=2 a=-c, n=1 / 2$ and there exists $i_{0} \in\{1, \ldots, n-1\}$ such that $a_{i 0} \neq$ 0 , or $b=2 a, n=1 / 2, G_{0}=a_{n}\left(x^{2}-2 a z\right)^{2 n-1}$, or $G_{0}=0$. Since $n$ is a natural number the two first cases are not possible, and therefore only the third case is possible. In this last case it is obvious that the Chen system has no polynomial first integral of the given form.

Case 2: $G_{0}$ has the form (10). Substituting $G_{0}$ into the second equation of (6) we get that

$$
\begin{aligned}
L\left[G_{1}\right]= & \sum_{i=0}^{n}[4 a i-2 c(n-i)] a_{i} \\
& \left(x^{2}-2 a z\right)^{2 i}\left(y^{2}+z^{2}\right)^{n-i} \\
+ & \sum_{i=0}^{n} 2\left[4 a^{2}-2 a b\right] i a_{i} \\
& \left(x^{2}-2 a z\right)^{2 i-1}\left(y^{2}+z^{2}\right)^{n-i} z \\
+ & \sum_{i=0}^{n} 2(b+c)(n-i) a_{i} \\
& \left(x^{2}-2 a z\right)^{2 i}\left(y^{2}+z^{2}\right)^{n-i-1} z^{2} .
\end{aligned}
$$

Then we have

$$
\begin{aligned}
\bar{G}_{1}= & \sum_{i=0}^{n} \frac{1}{a}[4 a i-2 c(n-i)] a_{i} \\
& u^{2 i} v^{n-i-1} \sqrt{u+2 a w} \sqrt{v-w^{2}} \\
+ & \sum_{i=0}^{n-1}\left(\frac{1}{a}[4 a i-2 c(n-i)] a_{i}+\right. \\
& \left.4 a(2 a-b)(i+1)] a_{i+1}\right) u^{2 i+1} v^{n-i-1} . \\
& \cdot \int \frac{w}{\sqrt{u+2 a v} \sqrt{v-w^{2}}} d w \\
+ & 4 a n a_{n} u^{2 n+1} v^{-1} \int \frac{w}{\sqrt{u+2 a v} \sqrt{v-w^{2}}} d w \\
+ & \sum_{i=0}^{n-1}[12 a i+2(b-2 c)(n-i)] a_{i} u^{2 i} v^{n-i-2} . \\
& \cdot \int \frac{w^{2}}{\sqrt{u+2 a v} \sqrt{v-w^{2}}} d w+\bar{T}_{2}(u, v) .
\end{aligned}
$$

In order that $G_{1}$ be a weight homogeneous polynomial of weight degree $4 n-1$, we must have $\bar{T}_{2}(u, v)=0$, and

$$
\begin{aligned}
& 4 a n a_{n}=0, \\
& (4 a i-2 c(n-i)) a_{i}+ \\
& 4 a^{2}(2 a-b)(i+1) a_{i+1}=0, \\
& (12 a i+2(b-2 c)(n-i)) a_{i}=0,
\end{aligned}
$$

with $i=0, \ldots, n-1$. We can easily prove that since $a \neq 0$, conditions (13) are equivalent to one of the 
following conditions:

$$
\begin{aligned}
b= & 2 a, n=0, a_{n} \neq 0, \text { and } \\
& a_{i}=0 \text { for } i=0,1, \ldots, n-1 \\
b= & 2 a=-c, n=0, \text { and there exists } \\
& i_{0} \in\{0, \ldots, n-1\} \text { such that } a_{i_{0}} \neq 0 \\
b= & -c \neq 2 a, 2 c n=0, a_{0} \neq 0, \text { and } \\
& a_{i}=0 \text { for } i=1, \ldots, n \\
b= & 6 a+2 c \neq-c, n=0, \\
& a_{n-i}\left(-4 a^{2}\right)^{i}\left(\begin{array}{c}
n \\
i
\end{array}\right) a_{n}, \text { and } a_{n} \neq 0 .
\end{aligned}
$$

Since $n \geq 1$ the unique possibility is $b=c=0$, $a \neq 0, a_{0} \neq 0$ and $a_{i}=0$ for $i=1, \ldots, n$. Since $G_{0}=a_{0}\left(y^{2}+z^{2}\right)^{n}$ and $G_{1}=0$, from (6) we get

$$
\begin{aligned}
G_{2}= & 2 a n a_{0}\left(y^{2}+z^{2}\right)^{n-1} z \\
& +\sum_{i=1}^{n} a_{i}^{(2)}\left(x^{2}-2 a z\right)^{2 i-1}\left(y^{2}+z^{2}\right)^{n-i} \\
= & a_{0}\left(\begin{array}{c}
n \\
1
\end{array}\right)\left(y^{2}+z^{2}\right)^{n-1}(2 a z) \\
& +\sum_{i=1}^{n} a_{i}^{(2)}\left(x^{2}-2 a z\right)^{2 i-1}\left(y^{2}+z^{2}\right)^{n-i} .
\end{aligned}
$$

Then again from (6) with $j=3$, we can prove that

$$
\begin{aligned}
\bar{G}_{3}= & \sum_{i=1}^{n}(2 a(2 i-1)) a_{i}^{(2)} u^{2 i-1} v^{n-i} . \\
& \cdot \int \frac{d w}{\sqrt{u+2 a w} \sqrt{v-w^{2}}} \\
& +4 a^{2} \sum_{i=1}^{n}(2 i-1) a_{i}^{(2)} u^{2 i-2} v^{n-i} . \\
& \cdot \int \frac{w}{\sqrt{u+2 a w} \int v-w^{2}} d w+\bar{T}_{3}(u, v) .
\end{aligned}
$$

In order that $G_{3}$ be a weight homogeneous polynomial of weight degree $4 n-3$, we must have $\bar{T}_{3}(u, v)=0$, and

$$
\begin{aligned}
& 2 a(2 i-1) a_{i}^{(2)}=0, \quad i=1, \ldots, n, \\
& 4 a^{2} a_{1}^{(2)}=0, \\
& 2 a^{2}(2 i-1) a_{i}^{(2)}=0, \quad i=2, \ldots, n .
\end{aligned}
$$

Consequently $G_{3}=0$. Indeed, from conditions (14) and since $a \neq 0$ is equivalent to $a_{i}^{(2)}=0$ for $i=$ $1, \ldots, n$, it follows that $G_{3}=0$. Hence

$$
G_{2}=a_{0}\left(\begin{array}{c}
n \\
1
\end{array}\right)\left(y^{2}+z^{2}\right)^{n-1}(2 a z) .
$$

Furthermore, substituting $G_{2}$ and $G_{3}$ in (6) with $j=4$, we get that

$$
G_{4}=a_{0} n(n-1)\left(y^{2}+z^{2}\right)^{n-2}(2 a)^{2} z+\sum_{i=0}^{n} a_{i}^{(4)} u^{2 i} v^{n-i-1} .
$$

Substituting $G_{3}$ and $G_{4}$ into (6) with $j=5$, we obtain that

$$
\begin{aligned}
\bar{G}_{5}= & 4 a i a_{i}^{(4)} u^{2 i} v^{n-i-1} \int \frac{1}{\sqrt{u+2 a w} \sqrt{v-w^{2}}} d w \\
& +\sum_{i=0}^{n} 8 a^{2} i a_{i}^{(4)} u^{2 i-1} v^{n-i-1} . \\
& \cdot \int \frac{w}{\sqrt{u+2 a w} \sqrt{v-w^{2}}} d w+\bar{T}_{5}(u, v) .
\end{aligned}
$$

Since $G_{5}$ must be a weight homogeneous polynomial of degree $4 n-5$, we must have $\bar{T}_{5}(u, v)=0$, and

$$
4 a i a_{i}^{(4)}=0, \quad 8 a i a_{i}^{(4)}=0, \quad i=0, \ldots, n .
$$

These equations imply that $i=0$ and $a_{i}^{(4)}=0$ for $i=1, \ldots, n$. Thus we have that $G_{5}=0$ and

$$
G_{4}=a_{0}\left(\begin{array}{c}
n \\
2
\end{array}\right)\left(y^{2}+z^{2}\right)^{n-2}(2 a)^{2} .
$$

By recursive calculations we can prove that

$$
\begin{aligned}
& G_{2 i}=a_{0}\left(\begin{array}{c}
n \\
i
\end{array}\right)\left(y^{2}+z^{2}\right)^{n-i}(2 a z)^{i}, \\
& G_{2 i+1}=0
\end{aligned}
$$

for $i \geq 0$ and $2 i \leq m$. Hence the polynomial first integral is

$$
\left(\begin{array}{c}
n \\
i
\end{array}\right)\left(y^{2}+z^{2}\right)^{n-i}(2 a z)^{i}=a_{0}\left(y^{2}+z^{2}+2 a z\right)^{n} .
$$

The generator of the polynomial first integral is $y^{2}+$ $z^{2}+2 a z$. This completes the proof of Theorem 1.2.

\section{Proof of Theorem 1.3}

In this section we prove Theorem 1.3. We introduce the weight change of variables in (3) and system (2) becomes

$$
\begin{aligned}
& X^{\prime}=a(Y-\mu X), \\
& Y^{\prime}=-X Z+\mu c Y, \\
& Z^{\prime}=X Y-\mu b Z,
\end{aligned}
$$

where again the prime denotes the derivative with respect to $T$. 
Let $g(x, y, z)$ be a polynomial first integral of system (2) and set $G(X, Y, Z)$ as in (4). We have

$$
\begin{aligned}
& a(y-\mu x) \sum_{i=0}^{m} \mu^{i} \frac{\partial G_{i}}{\partial x}+(-x z+\mu c y) \sum_{i=0}^{m} \mu^{i} \frac{\partial G_{i}}{\partial y} \\
& +(x y-\mu b z) \sum_{i=0}^{m} \mu^{i} \frac{\partial G_{i}}{\partial z}=0,
\end{aligned}
$$

where again we still use $x, y, z$ instead of $X, Y, Z$.

From the previous equality the coefficients of $\mu^{i}$ for $i=0,1, \ldots, m+1$ are

$$
\begin{aligned}
L\left[G_{0}\right] & =0, \\
L\left[G_{j}\right] & =a x \frac{\partial G_{j-1}}{\partial x}-c y \frac{\partial G_{j-1}}{\partial y}+b z \frac{\partial G_{j-1}}{\partial z},
\end{aligned}
$$

for $j=2,3, \ldots, m+1$, where $G_{j}=0$ for $j>m$ and $L$ is the linear partial differential operator in (7). Proceeding as in the proof of Theorem 1.2 we get that $G_{0}$ has either the form (10) with weight degree $4 m$, or the form (11) with weight degree $4 m-2$. Proceeding analogously to Case 1 in the proof of Theorem 1.2 we get that the Lü system has no polynomial first integrals if $G_{0}$ is of the form (11). Again, proceeding as in Case 2 of the proof of Theorem 1.2 we get that $G_{1}=0$ and again from (13) and since $a \neq 0$, the unique possibility is $b=c=0$, $a_{0} \neq 0$ and $a_{i}=0$ for $i=1, \ldots, n$. Therefore $G_{0}=a_{0}\left(y^{2}+z^{2}\right)^{n}$, and

$$
\begin{aligned}
G_{2} & =\sum_{i=1}^{n} a_{i}^{(2)}\left(x^{2}-2 a z\right)^{2 i-1}\left(y^{2}+z^{2}\right)^{n-i} \\
& =\sum_{i=1}^{n} a_{i}^{(2)}\left(x^{2}-2 a z\right)^{2 i-1}\left(y^{2}+z^{2}\right)^{n-i} .
\end{aligned}
$$

Then from (15) with $j=3$, and proceeding as in the proof of Theorem 1.2 we get that $a_{i}^{(2)}=0$ for $i=1, \ldots, n$, and consequently $G_{2}=G_{3}=0$. By recursive calculations, and proceeding as in Case 2 in the proof of Theorem 1.2 we can prove that $G_{i}=0$ for $i=1, \ldots, n$. Hence the generator of the polynomial first integrals is $y^{2}+z^{2}$. This completes the proof of Theorem 1.3.

\section{Acknowledgements}

The first author is partially supported by the MICIIN/FEDER grant MTM2008-03437, the Generalitat de Catalunya grant 2009SGR-410 and ICREA Academia. The second author is partially supported by FCT through CAMGDS, Lisbon.

\section{References}

Bashkirtseva, I., Chen, G., \& Ryashko, L., [2010] "Analysis of stochastic cycles in the Chen system," Internat. J. Bifur. Chaos Appl. Sci. Engrg. 20, 1439-1450.

Cafagna, D., \& Grassi, G.Y., [2008] "Bifurcation and chaos in the fractional-order Chen system via a time-domain approach," Internat. J. Bifur. Chaos Appl. Sci. Engrg. 18, 1845-1863.

Cai, N., Jing, Y.W., \& Zhang, S.Y., [2009] "Antisynchronization between hyper-chaotic Chen system and hyper-chaotic Lorenz system," $J$. Northeast. Univ. at. Sci. 30, 313-317.

Cao, J., Chen, C., \& Zhang, X., [2008] "The Chen system having an invariant algebraic surface," Internat. J. Bifur. Chaos Appl. Sci. Engrg. 18, 3753-3758.

Chen, G., \& Ueta, T., [1999] "Yet another chaotic attractor," Internat. J. Bifur. Chaos Appl. Sci. Engrg. 9, 1465-1466.

Chen, L., \& Wang, D.S., [2007] "Adaptive tracking control of the Chen system," Acta Phys. Sinica 56, 5661-5664.

Chen, T., \& Zhou, S.F., [2009] "Synchronization in lattices of coupled non-autonomous Chen system via Lyapunov function," J. Shanghai Univ. 13, 242-247.

Chowdhury, M.S., \& Hashim, I., [2009] "Application of mutistage homotopy-perturbation method for the solutions of the Chen system," Nonlinear Anal. Real World Appl. 10, 381-391.

Darboux, G., [1878] "Mémoire sur les équations différentielles du primer ordre et du premier degrée (Mélanges)," Bull. Sci. Math. 2éme Série 2, 60-96, 123-144, 151-200.

Denquan, L., \& Zhixiang, Y., [2009] "Connecting the Lorenz and Chen systems via nonlinear control," Commun. Nonlinear Sci. Numer. Simul. 14, 655-667.

Fallahi, K., Raoufi, R., \& Khoshbin, H., [2008] "An application of Chen system for secure chaotic 
communication based on extended Kalmar filter and multi-shift cipher algorithm," Commun. Nonlinear Sci. Numer. Simul. 13, 763-781.

Forsyth, A.R., [1900] Theory of differential Equations Cambridge University Press, Cambridge.

Hou, Z., Kang, N., Kong, X., \& Chen, G., [2008] "On the equivalence of Lorenz system and Chen system," Internat. J. Bifur. Chaos Appl. Sci. Engrg. 13, 763-781.

Jouanolou, J.P., [1979] Equations de Plaff algébriques Lecture Notes in Mathematics 708 Springer, New York.

Llibre, J., \& Zhang, X., [2002] "Invariant algebraic surfaces of the Lorenz system," J. Math. Phys. 43, 1622-1645.

Lü, T., [2007] "Exponential factors and nonDarbouxian integrability of the Chen system," Ann. of Diff. Eqs. 23, 304-311.

Lü, T., [2009] "Darboux polynomials and nonalgebraic integrability of the Lü system," Ann. Differential Equations 25, 420-431.

Lü, J. \& Chen, G., [2002] "A new chaotic attractor coined," Internat. J. Bifur. Chaos Appl. Sci. Engrg. 12, 659-661.

Lü, T. \& Zhang, X., [2007] "Darboux polynomials and algebraic integrability of the Chen system," Internat. J. Bifur. Chaos Appl. Sci. Engrg. 17, 2739-2748.

Mahmoud, G.M., Mahmoud, E.E., \& Ahmed, M.E., [2007] "A hyperchaotic complex Chen and its dynamics," Internat. J. Appl. Math. Stat. 12, 90100.

Poincaré, H., [1891] "Sur l'intégration des équations différentielles du primer ordre et du primer degreée I," Rendicontin del Circolo Matematico di Palermo 5, 161-191.

Yao, H., \& Liu, Z., [2010] "The application of contraction theory in synchronization of coupled Chen systems," Int. J. Nonlinear Sci. 9, 72-77. 